\title{
Delta opioid receptors on nociceptive sensory neurons mediate peripheral endogenous analgesia in colitis
}

\author{
Xavier Mas-Orea', Lilian Basso ${ }^{1,2}$, Catherine Blanpied ${ }^{1}$, Claire Gaveriaux-Ruff ${ }^{3}$, Nicolas Cenac ${ }^{1}$ and \\ Gilles Dietrich ${ }^{1 *}$ (1)
}

\begin{abstract}
Background: Inflammatory visceral pain is endogenously controlled by enkephalins locally released by mucosal $\mathrm{CD}^{+}$T lymphocytes in mice. The present study aimed at identifying opioid receptor(s) expressed on nociceptive sensory nerves involved in this peripheral opioid-mediated analgesia.

Methods: The peripheral analgesia associated with the accumulation of $\mathrm{CD}_{4}^{+} \mathrm{T}$ lymphocytes within the inflamed colonic mucosa was assessed in conditional knockout mice specifically deleted for either of the two opioid receptors for enkephalins (i.e., $\mu$ (MOR) and $\delta$ (DOR) receptors) in $\mathrm{Na}_{v}$ 1.8-expressing sensory neurons in the dextran sulfate sodium (DSS)-induced colitis model.

Results: Endogenous analgesia is lost in conditional knockout mice for DOR, but not MOR at the later phase of the DSS-induced colitis. The absence of either of the opioid receptors on sensory nerves had no impact on both the colitis severity and the rate of T lymphocytes infiltrating the inflamed colonic mucosa.

Conclusion: The key role of DOR on primary afferents in relieving intestinal inflammatory pain opens new therapeutic opportunities for peripherally restricted DOR analgesics to avoid most of the side effects associated with MORtargeting drugs used in intestinal disorders.
\end{abstract}

Keywords: Visceral pain, Opioid receptors, Nociceptors, T lymphocytes, Opioids, Intestinal inflammation

\section{Background}

Management of non-cancer chronic pain with opioids has not any long-term benefits as dose escalation, associated with opioid tolerance, often results in addiction and overdose death $[39,47]$. It has been estimated that $21 \%$ of outpatients with inflammatory bowel disease (IBD) are opioid users [30] and that 5\% turn into heavy users [43]. In addition, opioid therapy induces intestinal adverse effects such as constipation and ileus that may

\footnotetext{
*Correspondence: gilles.dietrich@inserm.fr

1 Digestive Health Research Institute (IRSD), Université de Toulouse,

INSERM, INRA, ENVT, UPS, CHU Purpan BP 3028, 31024 Toulouse Cedex 3, France

Full list of author information is available at the end of the article
}

lead to narcotic bowel syndrome, which paradoxically, makes pain worse, thereby leading patients into a vicious cycle of addiction. To limit opioid use disorders, a variety of strategies have been proposed including biased ligands [28, 38] or ligands specific for receptor splice-variant [27]. Because these approaches only partially overcome centrally mediated side effects such as addiction, alternative strategies based on the endogenous mechanisms of pain regulation in periphery are under investigation $[8$, $14,26,41]$. They consist in locally sustaining the endogenous opioid activity $[13,35]$ or in activating peripheral opioid receptors $[15,25]$ that may be targeted only in an acidic inflammatory environment $[2,20,23,37,40]$. In this context, the finding that endogenous opioids locally 
produced by mucosal $\mathrm{CD} 4^{+} \mathrm{T}$ lymphocytes $[4,9-11,44-$ 46] alleviate inflammation-induced visceral pain represents an opportunity to improve opioid therapy. Beyond the potential therapeutic use of effector memory $\mathrm{T}$ lymphocytes including active vaccination $[1,5]$ or passive $\mathrm{T}$ cell transfer (immunotherapy) [24, 49], a better knowledge of the opioid receptor(s) involved in the peripheral analgesic effects of $\mathrm{T}$ lymphocytes might also refine prescription of opioid medications.

In this study, we compared the visceral sensitivity of mice with opioid receptor-deficient nociceptors to that of control opioid receptor floxed mice in the dextran sulfate sodium (DSS)-induced colitis model in which T lymphocyte accumulation induces analgesia $[5,9,10,23,46]$.

\section{Methods}

\section{Animals and experimental colitis}

The MOR-floxed $\left(O p r m 1^{\mathrm{fl} / \mathrm{fl}}\right)$ and DOR-floxed (Oprd1 $1^{\mathrm{fl} /}$

fl) mouse lines were crossed with $\mathrm{Na}_{v} 1.8$-Cre mice to produce conditional knockout (cKO) mice for MOR and DOR in afferent nociceptive neurons, respectively $[16,48]$. All mice were on a mixed genetic background (C57BL6/J-SV129Pas) and were bred in the animal care facility at Toulouse (INSERM US 006 ANEXPLO/CREFRE, Toulouse, France).

Polymerase chain reaction (PCR) genomic DNA analyses were performed on DNA from dorsal root ganglia (DRG) neurons and brain to confirm Oprm1 exon 2 and 3 deletion and Oprd1 exon 2 deletion in DRGs and not the brain in cKO MOR and cKO DOR mice, respectively, as previously described $[16,48]$. The forward primers A (5'-ACCAGTACATGGACTGGATGTGCC-3') and C (5'-GTTACTGGAGAATCCAGGCCAAGCC-3') and the reverse primers $B$ (5'-TGCTAGAACCTGCGGAGC CACA-3') and D (5'-CGCTTGGGAATATCTTGTACC TATGACCA-3') were used to reveal Oprm1 gene excision and intact floxed allele, respectively [48]. Oprd1 gene excision and intact floxed allele were detected by using the forward primer E (5'-GGTTAGCCTTCTGAG GGCTGGG-3') and the reverse primer $\mathrm{F}$ (5'-CCTGGC CAGCCAGTTCACAATCT-3').

Mice used in the study were 8- to 12 -week-old males weighing $20-25 \mathrm{~g}$. They were housed by four in ventilated cages at a temperature between 20 and $22{ }^{\circ} \mathrm{C}$ and maintained under a $12 \mathrm{~h} \mathrm{light/dark} \mathrm{cycle} \mathrm{in} \mathrm{sawdust-coated}$ transparent cages with chow and water ad libitum. Controls were MOR or DOR-floxed littermate mice that did not express the Cre recombinase.

Colitis was induced by adding 3\% (weight/volume) dextran sulfate sodium (DSS, 36,000-50,000 MW; MP Biomedicals, Illkirch, France) into the drinking water for 5 days. Then, from day 5 until day 12, animals received only water as previously described [10]. All procedures involving animals were performed in accordance with the Guide for the Care and Use of Laboratory Animals of the European Council and were approved by the Animal Care and Ethics Committee of US006/CREFE (CEEA-122; application number APAFIS \#16385-2018080222083660 v3).

\section{Real-time quantitative RT-PCR analysis}

Brain and DRG samples were homogenized in $500 \mu \mathrm{L}$ TRIzol $^{\text {TM }}$ Reagent (Sigma). Total RNA was then isolated by using GenElute ${ }^{\mathrm{TM}}$ Mammalian total RNA miniprep Kit following the manufacturer's instructions (Sigma). RNA was evaluated using a ND-1000 Nanodrop spectrophotometer and gel electrophoresis. RNA was then reverse-transcribed with Moloney murine leukemia virus reverse transcriptase using random hexamers for priming. Transcripts encoding hypoxanthine phosphoribosyl transferase (HPRT), $\mathrm{Na}_{\mathrm{v}} 1.8, \mathrm{MOR}$ and DOR were quantified by real-time quantitative polymerase chain reaction using the following forward and reverse primers: 5'-CAT TGCTGACAGGATGCAGAAGG-3' and 5'- TGCTGG AAGGTGGACAGTGAGG-3' for $\beta$-actin, 5 '- ATCGAA GCCAAGGAGAAGAAGTT-3' and 5'-CTGCCAGCA CCTCCTGTT-3' for SCN10A ( $\left.\mathrm{Na}_{\mathrm{v}} 1.8\right)$, 5'- GAGCCA CAGCCTGTGCCCT-3' and 5'-CGTGCTAGTGGCTAA GGCATC-3' for Oprm1, 5'-GCTCGTCATGTTTGG CATC-3' and 5'-AAGTACTTGGCGCTCTGGAA-3' for Oprd1. The target gene expression was normalized to that of the $\beta$-actin and the $2^{-\Delta \Delta C T}$ method was used to evaluate mRNA expression levels relative to the corresponding littermate floxed control animals.

\section{Macroscopic assessment of colon injury}

Macroscopic damage was evaluated using a scale ranging from 0 to 11: erythema (absent (0), length of the area less than $1 \mathrm{~cm}(1)$, more than $1 \mathrm{~cm}(2))$, edema (absent (0), mild (1), severe (2)), strictures (absent (0), one (1), two (2), more than two (3)), ulceration (absent (0), present (1)), mucus (present (0), absent (1)), and adhesion (absent (0), moderate (1), severe (2)). Bowel wall thickness was measured with an electronic calliper at $0.5 \mathrm{~cm}$ above the anus (colorectum).

\section{Histological assessment of colon injury}

Colonic tissue specimens were excised $0.5 \mathrm{~cm}$ proximal to the anus. Damage scoring was evaluated on $5-\mu \mathrm{m}$ colonic sections stained with haematoxylin-eosin using a scale ranging from 0 to 12 . Inflammatory cell infiltration, epithelial/mucosal alteration (including vasculitis, goblet cell depletion and crypt abscesses), mucosal architecture alteration (including ulceration and crypt loss) and submucosal edema were graded from 0 to 3 (absent, mild, moderate and severe). 


\section{Assessment of mucosal lymphocyte density}

Five-micrometer colonic sections deparaffinized, rehydrated and saturated with PBS 1\% BSA were incubated with rabbit anti-CD3 (Clone SP7, Diagnostic BioSystems, Pleasanton, CA) monoclonal antibody for $1 \mathrm{~h}$ at room temperature. After washing with PBS, bound antibodies were revealed by further incubation with Alexa Fluor 555-labeled Donkey anti-rabbit IgG antibodies (Life Technologies, Carlsbad, CA). Slides were mounted and nuclei were stained with 4,6-diamidino-2-phenylindole (DAPI) fluorescent mounting medium (ProLong ${ }^{\mathrm{TM}}$ Gold, Invitrogen). Fluorescence images were taken using ApoTome Zeiss Axio-observer (Carl Zeiss Microscopy $\mathrm{GmbH}$, Iéna, Allemagne) with $\times 20$ objective. Colonic density of $\mathrm{T}$ lymphocytes was determined by counting anti-CD3 fluorescence cells relative to tissue surface delimited by using DAPI staining thanks to an algorithm running on Fiji software [10].

\section{Visceral sensitivity assessment by colorectal distension}

Visceral sensitivity was examined by measuring the visceromotor response to colorectal distension. Three days before colorectal distension, 2 electrodes (Bioflex insulated wire AS631; Cooner Wire, Chatsworth, CA) were implanted into the abdominal external oblique musculature of mice previously anaesthetized with ketamine $\left(10 \mathrm{mg} \mathrm{kg}^{-1}\right)$ and xylazine $\left(1 \mathrm{mg} \mathrm{kg}^{-1}\right)$. Electrodes were exteriorized at the back of the neck and protected by a plastic tube attached to the skin. After surgery, mice were examined twice a day for abnormal behavior (absence of grooming, immobility) until the day of the distension. On the day of the distension, electrodes were connected to a Bio Amp, itself connected to an electromyogram acquisition system (ADInstruments, Inc., Colorado Springs, $\mathrm{CO})$. Ten-second distensions were performed on conscious animals with a $10.5-\mathrm{mm}$-diameter balloon catheter (Fogarty catheter for arterial embolectomy, 4F; Edwards Lifesciences, Nijmegen, Netherlands) gently inserted into the colorectum at $5 \mathrm{~mm}$ from the anus (balloon covering a distance of $1 \mathrm{~cm}$ starting at $5 \mathrm{~mm}$ from the anus) and progressively inflated at pressures of $15,30,45$ and $60 \mathrm{~mm} \mathrm{Hg}$ with 5-min rest intervals. Electromyography activity of abdominal muscles was recorded to calculate visceromotor responses using LabChart 8 software (ADinstruments) [34].

\section{Statistical analysis}

Data are expressed as mean \pm SEM. The comparison between two groups of animals was performed using Mann-Whitney U test. Kruskal-Wallis test was used to compare three groups of mice. Comparison of visceral sensitivity in response to increasing distension pressures between animals were estimated with two-way repeated measurements analysis of variance (ANOVA). All statistical analyses were performed by using Prism 9.0 statistical software (GraphPad, San Diego CA).

\section{Results}

To identify receptor(s) responsible for the opioid-induced analgesia at the later phase of the DSS-induced colitis, we used conditional knockout (cKO) mice in which the enkephalin opioid receptors mu (MOR) or delta (DOR) are specifically deleted in $\mathrm{Na}_{\mathrm{v}} 1.8$-expressing nociceptive neurons. cKO mice in which MOR (Oprm1) or DOR (Oprd1) genes are specifically deleted in nociceptors were generated by crossing $O p r m 1^{\mathrm{f} / \mathrm{fl}}$ or $O p r d 1^{\mathrm{f} / \mathrm{fl}}$ mice with mice that express Cre recombinase in $\mathrm{Na}_{\mathrm{v}} 1$.8-expressing neurons $[16,48]$. As genetic background may impact pain assays, the cKO MOR or cKO DOR were compared to their respective Cre-negative MOR flox or DOR flox littermates with the same genetic background. As shown in Fig. 1, the cKO MOR or cKO DOR mouse lines are specifically deleted for MOR (Fig. 1A) or DOR (Fig. 1F) in peripheral nociceptive neurons but not in other tissue as brain, thereby ruling out a non-specific germline activation of the Cre recombinase. The absence of any alteration of $\mathrm{Na}_{\mathrm{v}} 1.8$ (SCN1OA) mRNA expression levels in both cKO MOR (Fig. 1B) and cKO DOR (Fig. 1G) suggested that the targeted deletion of the opioid receptor genes did not impact $\mathrm{Na}_{\mathrm{v}} 1.8$ sodium channels in nociceptors. The two cKO mouse strains exhibited virtually similar 53-54\% reduction of the targeted opioid receptors MOR (Fig. 1C) or DOR (Fig. 1H) in DRG neurons as assessed by quantitative PCR. The specific deletion of MOR in DRGs did not modify the expression of DOR (Fig. 1D), while the specific deletion of DOR was associated with a weak but significant up-regulation of MOR in DRGs (Fig. 1 I). The similar expression levels of MOR (Fig. 1E) and DOR (Fig. 1J) mRNA in the brain of the cKO mice as compared to their floxed control littermates confirmed the absence of non-specific germline activation of the Cre recombinase in each cKO mouse strain.

We then confirmed that the MOR and DOR-floxed control littermates with a C57BL6/J-SV129Pas genetic background responded to DSS treatment as already shown in BALB/c mice [10]. As expected, after five consecutive days of treatment with $3 \%$ DSS, floxed mice developed colitis characterized by a significant reduction of colon length, an increase of wall thickness as well as macroscopic and microscopic tissue injuries (Fig. 2A-E) while $\mathrm{T}$ lymphocyte density within the mucosa remained stable as compared to untreated mice (Fig. 2F). This early phase of the disease was associated with visceral hypersensitivity (Fig. 3). As compared to mice treated 5 days with 3\% DSS (Day 5), those receiving water for 7 


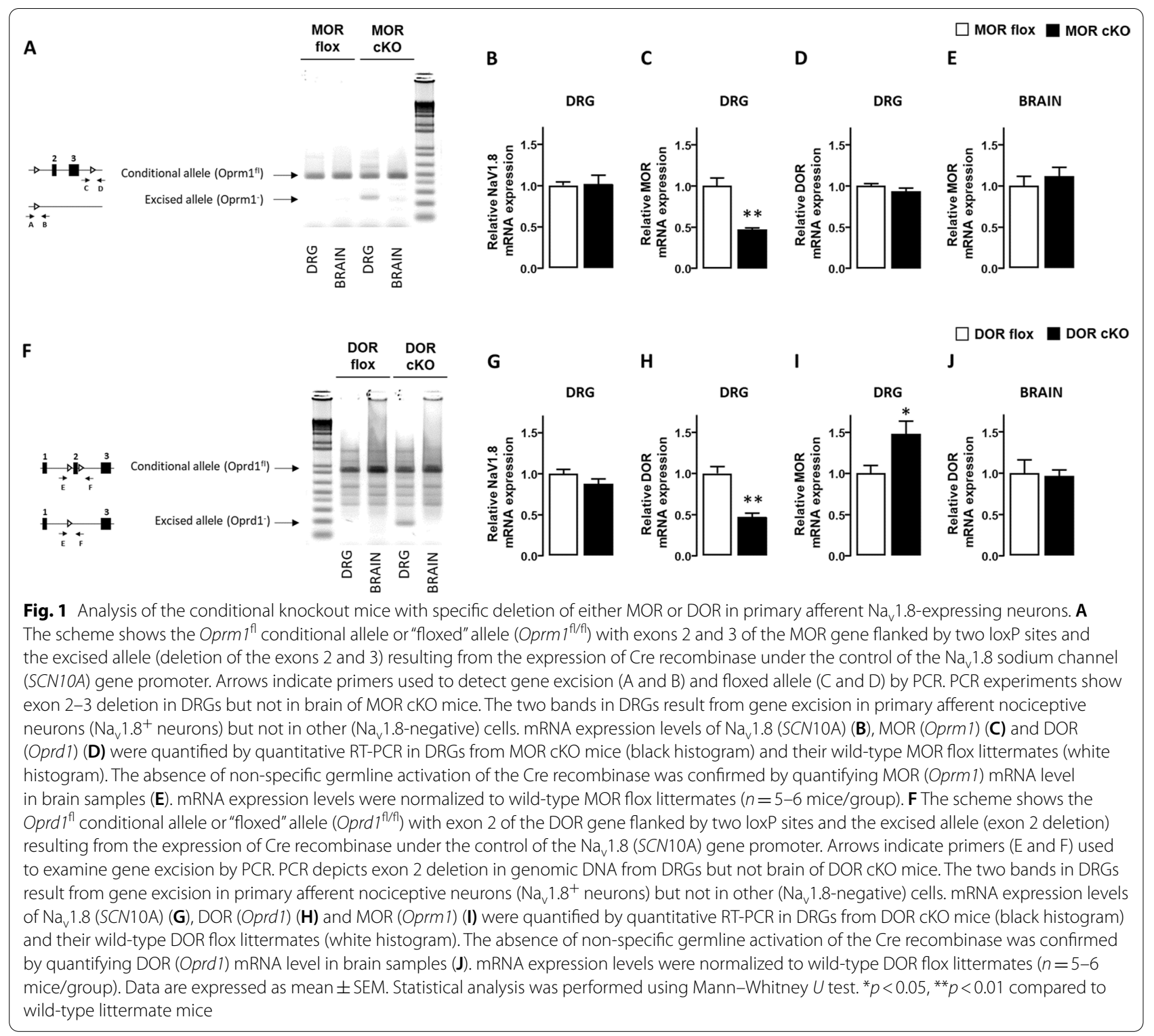

additional days (Day 12) showed a significant increase in mucosal T lymphocyte density (Fig. 2F). Despite colitisassociated damage at day 12 of the DSS treatment, the increase in lymphocytes within the inflamed lamina propria was associated with a normalization of the visceral sensitivity as shown by the abdominal response to colorectal distension similar between DSS-treated and untreated mice (Fig. 3).

To identify which opioid receptor on nociceptors is involved in the spontaneous analgesic effect observed in the later phase of the disease, we compared conditional MOR or DOR knockout mice to their floxed control littermates on day 12 of the DSS-induced colitis. We first determined whether MOR and DOR conditional knockout mice differ or not from their floxed littermates for colitis parameters. As shown in Fig. 4, the deletion of MOR or DOR in $\mathrm{Na}_{\mathrm{v}} 1.8^{+}$sensory nerves did not alter neither the severity of colitis (Fig. 4A-E and H-L) nor the accumulation rate of $\mathrm{T}$ lymphocytes within the inflamed mucosa (Fig. 4F-G and M, N). The impact of the deletion of each opioid receptor on the sensitivity of mice to colorectal distension was then investigated at day 12 of the DSS-induced colitis. Whereas the loss of MOR in nociceptors had no effect on the visceral sensitivity, that of DOR resulted in the loss of analgesia (Fig. 5). To confirm that the activation of DOR was dependent on effector $\mathrm{T}$ lymphocytes infiltrating the inflamed mucosa, we then assessed DOR conditional knockout mice at 


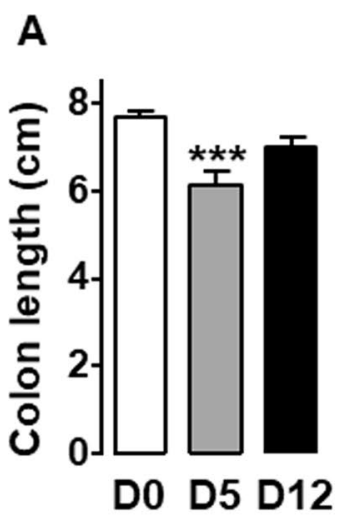

D

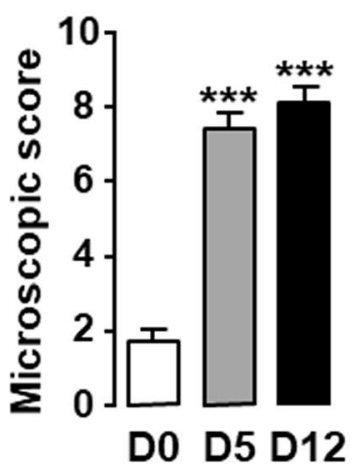

$\mathbf{F}$
B

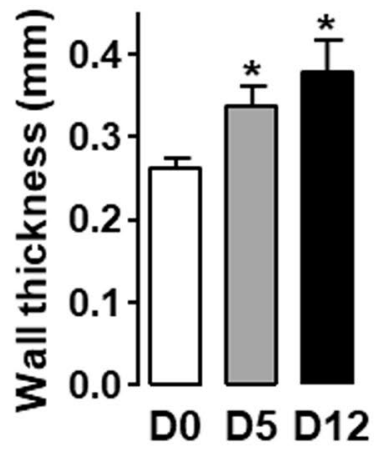

E
C

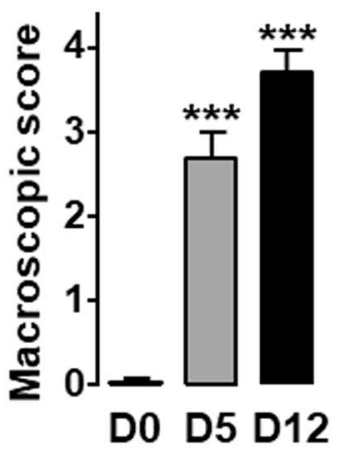

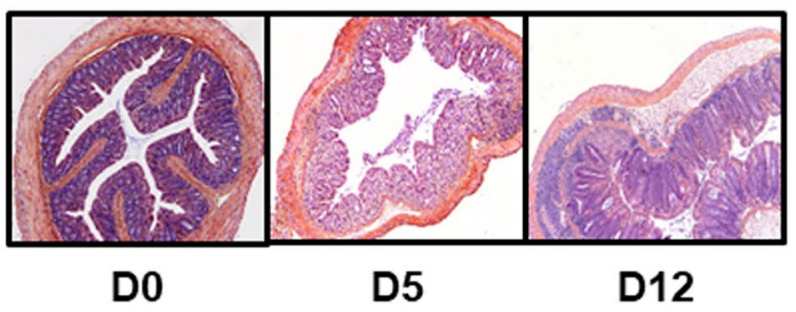

G

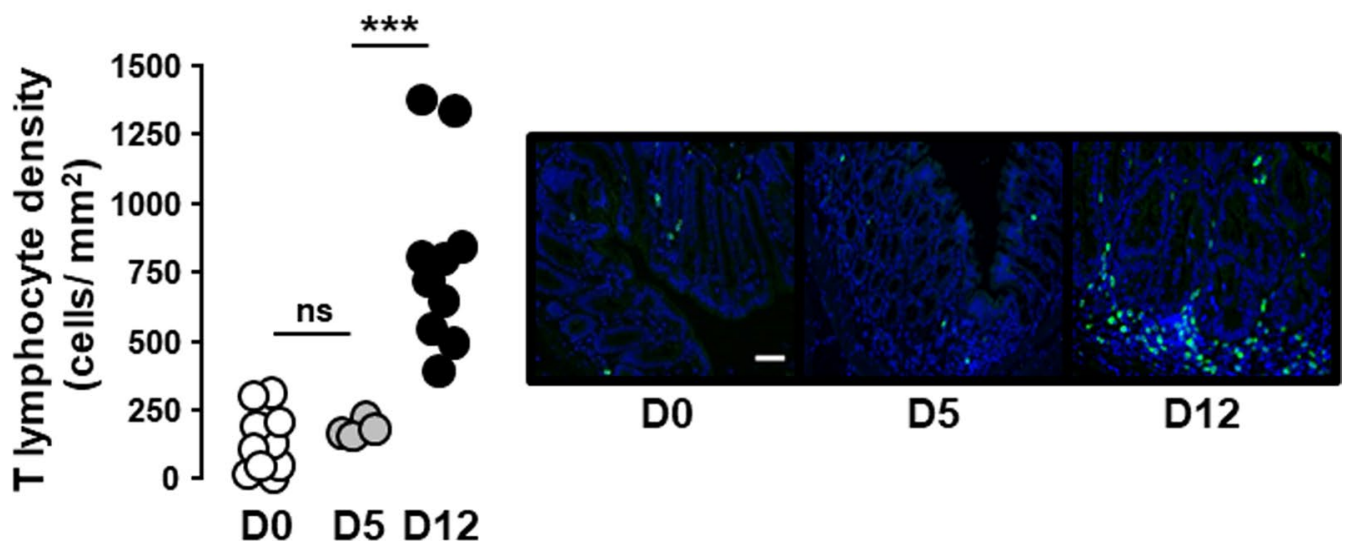

Fig. 2 T lymphocytes accumulate within inflamed colonic mucosa upon DSS-induced colitis. Littermate wild-type mice untreated (D0, white), treated 5 days with 3\% DSS (D5, grey) or treated with 3\% DSS for 5 days and then with water until day 12 (D12, black) were examined for colitis severity including colonic length (A), wall thickness (B), macroscopic (C) and histological (D) colonic tissue damage ( $n=10-20$ mice/group). A representative histopathological analysis performed on H\&E-stained colon sections in depicted in $\mathbf{E}$. Untreated mice (D0) show normal epithelial architecture with neither cellular infiltration nor edema (E, left panel). Mice treated 5 days with 3\% DSS (D5) show massive cellular infiltration, submucosal edema and epithelial architecture disruption (E, middle panel). Mice treated with 3\% DSS for 5 days and then with water until day 12 (D12) show massive cellular infiltration, severe edemas (submucosal and subepithelial) and epithelial disruption (E, right panel). $\mathbf{F}$ Density of CD3 ${ }^{+}$ T lymphocytes within lamina propria (each point, corresponding to one animal, represents the mean of four different histological examinations per slice) quantified per $\mathrm{mm}^{2}$ of tissue ( $n=4$ (D5) or 10 (D0 and D12) mice/group). A representative anti-CD3 immunostaining at day 0 (left panel), day 5 (middle panel) and day 12 (right panel) of the treatment is depicted in $\mathbf{G}$ (scale $50 \mu \mathrm{m}$ ). Data are expressed as mean \pm SEM. Statistical analysis was performed using Kruskal-Wallis and subsequent Dunn's multiple comparison tests. ${ }^{*} p<0.05,{ }^{* * *} p<0.001$ compared to normal control mice (D0) 


\section{A $O$ Day $0 \bigcirc$ Day $5 \bigcirc$ Day 12}

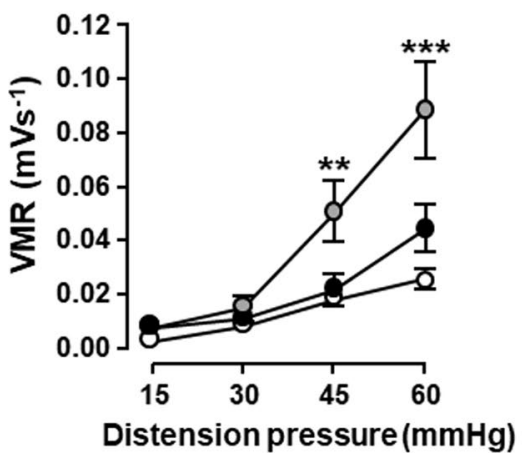

B

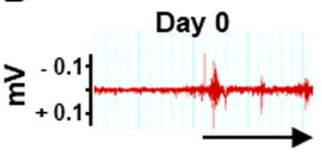

Day 5

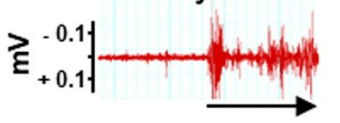

Day 12

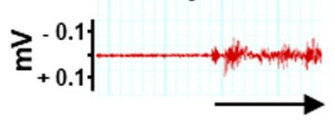

C

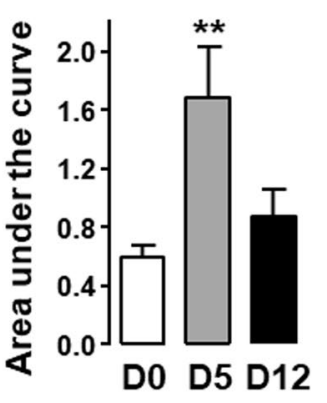

Fig. 3 Visceral sensitivity normalizes on day 12 of the DSS-induced colitis. Visceral sensitivity was examined in littermate wild-type mice untreated (Day 0, white), treated 5 days with 3\% DSS (Day 5, grey) or treated with 3\% DSS for 5 days and then with water until day 12 (Day 12, black) by measuring visceromotor response (VMR) to colorectal distension ( $n=9-18$ mice/group) (A). B Representative traces of abdominal muscle contractions in response to $60 \mathrm{~mm} \mathrm{Hg}$ colorectal distension in mice at day 0 (upper panel), day 5 (middle panel) and day 12 (lower panel) of the treatment. The arrows represent the $10 \mathrm{~s}$ of distension. Area under the curve (AUC) calculated by plotting individual VMR is shown in C. Data are expressed as mean \pm SEM. Statistical analysis was performed using repeated-measures two-way ANOVA and subsequent Sidak's multiple comparison tests (A) or Kruskal-Wallis and subsequent Dunn's multiple comparison tests $(\mathbf{C}) .{ }^{* *} p<0.01,{ }^{* * *} p<0.001$ compared to normal control mice (D0)

the early phase of the disease. As depicted in Fig. 6, the deletion of DOR in $\mathrm{Na}_{\mathrm{v}} 1.8^{+}$sensory neurons, as that of the MOR, did not affect the visceral hypersensitivity observed at day 5 of the DSS treatment. This latter result was observed when $\mathrm{T}$ lymphocytes did not yet infiltrate the mucosa (Fig. 6C and F), in agreement with our previous study [36]. It supports the pivotal role of DOR expressed in $\mathrm{Na}_{\mathrm{v}} 1.8^{+}$nociceptors in the $\mathrm{T}$ lymphocytesmediated endogenous control of inflammatory visceral pain.

\section{Discussion}

$\mathrm{T}$ lymphocytes are key regulators of inflammatory visceral pain as exemplified in the dextran sulfate sodium (DSS)-induced colitis model [5, 6, 10, 46]. In this model, intestinal epithelium integrity disruption by DSS leads to the translocation into the mucosa of bacteria that first activate innate immune cells before $\mathrm{T}$ cell-mediated adaptive immune response takes place few days later. The two steps of the immune response match with the painful (acute) and painless (later) phases of the disease. In this colitis model, enkephalins locally released by $\mathrm{T}$ lymphocytes infiltrating the inflamed mucosa on the later phase of the disease normalize visceral sensitivity by acting on opioid receptors expressed in nociceptor endings $[5,7$, 10].

Here, our study shows that peripheral DOR plays a major role in the endogenous regulation of inflammatory visceral pain in mice. Indeed, the targeted deletion of DOR in $\mathrm{Na}_{\mathrm{v}} 1$.8-expressing nociceptors prevents from the endogenous opioid-mediated analgesia at the latter phase of the DSS-induced colitis when T lymphocytes accumulate within the inflamed mucosa. Conditional knockout mice for MOR and DOR which are heterozygous for the Cre recombinase into the $\mathrm{Na}_{\mathrm{v}} 1.8$ locus preserve the expression of $\mathrm{Na}_{\mathrm{v}} 1.8$ channels in nociceptive neurons [42]. Accordingly, heterozygous $\mathrm{Na}_{\mathrm{v}} 1.8$-Cre recombinase mice display normal nociceptive inflammatory response [29]. Among the colonic afferents, MOR and DOR mRNA were found, respectively, in $38 \%$ and $46 \%$ of

\footnotetext{
(See figure on next page.)

Fig. 4 MOR or DOR deletion in $\mathrm{Na}_{v}$ 1.8-expressing sensory neurons does not alter neither colitis severity nor T cell infiltration within inflamed mucosa. Conditional knockout mice (black histogram) for either MOR (MOR cKO) (A-G) or DOR (DOR CKO) (H-N) were compared to their corresponding littermate wild-type floxed mice (MOR flox and DOR flox, respectively) (white histogram) in the basal conditions (Day 0) and on day 12 of DSS treatment for colitis severity ( $n=7-8$ mice/group) $\left(\mathbf{A}-\mathbf{E}\right.$ and $\mathbf{H}-\mathbf{L}$ ) and mucosal CD3 ${ }^{+}$T lymphocyte density (each point, corresponding to one animal, represents the mean of four different histological examinations per slice) ( $\mathbf{F}, \mathbf{G} ; n=10$ mice/group and $\mathbf{M}, \mathbf{N} ; n=6-14$ mice/group). Representative histopathological H\&E-stained colon sections (E and $\mathbf{L}$ ) and anti-CD3 immunostaining ( $\mathbf{G}$ and $\mathbf{N}$ ) (scale $50 \mu \mathrm{m}$ ) at day 0 and day 12 of the DSS treatment for conditional MOR $(\mathbf{E}, \mathbf{G})$ and DOR $(\mathbf{L}, \mathbf{N})$ KO mice and their respective littermate wild-type MOR and DOR-floxed mice are shown. Data are expressed as mean \pm SEM. Statistical analysis was performed using Mann-Whitney $U$ test
} 


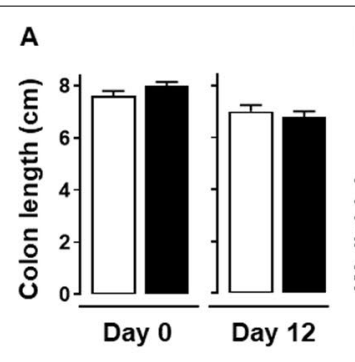

D

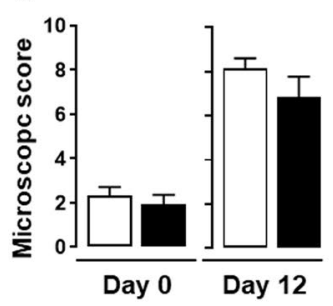

$F$

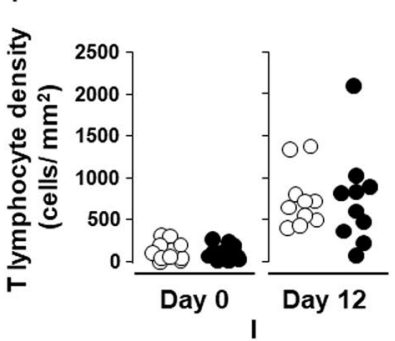

H

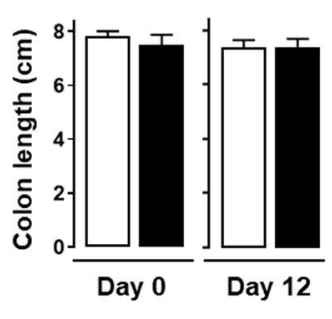

K
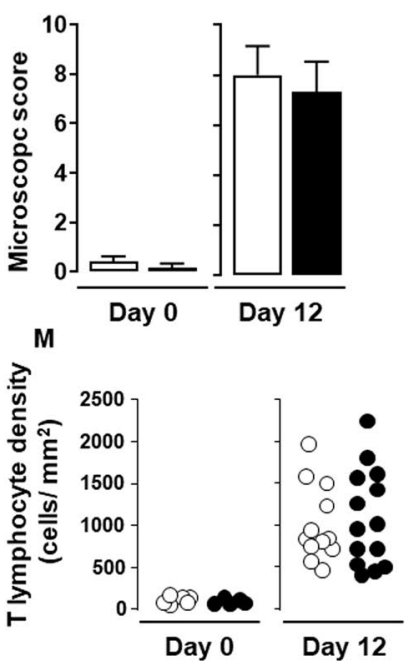

B C

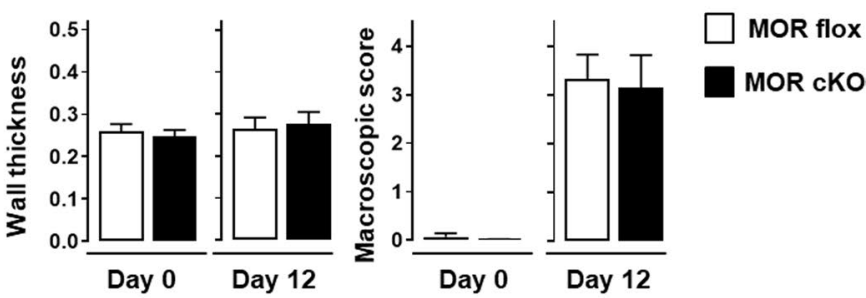

E

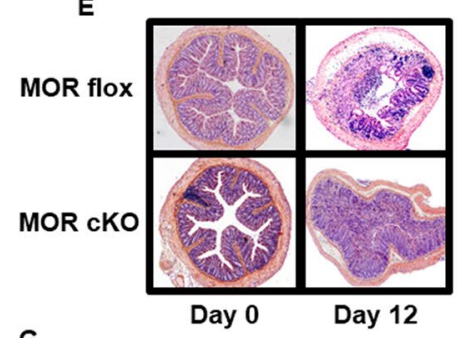

G

MOR flox

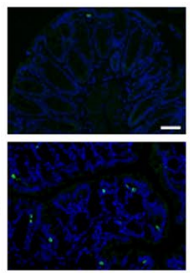

Day 0

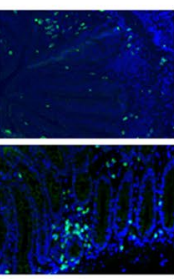

Day 12

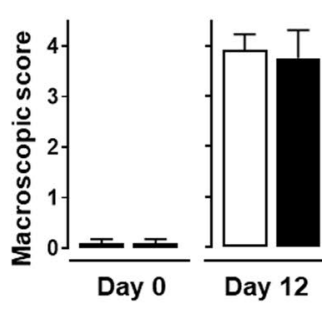

DOR flox

DOR cKO

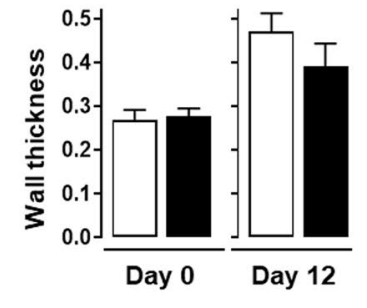

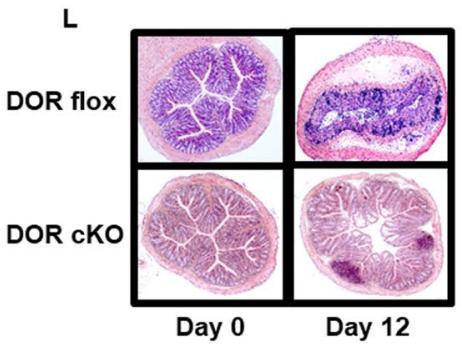

$\mathbf{N}$

DOR flox
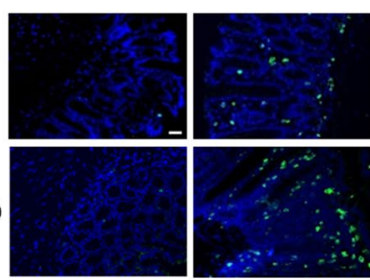

Day 12 


\section{A O MOR flox MOR cKO}

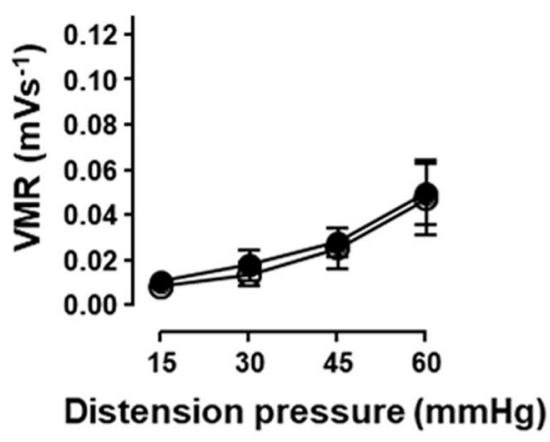

\section{O DOR flox - DOR cKO}

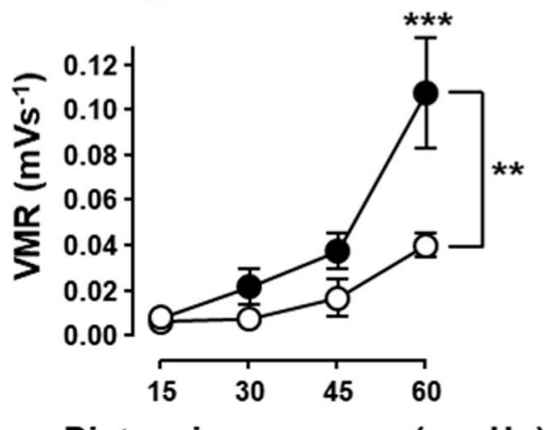

B

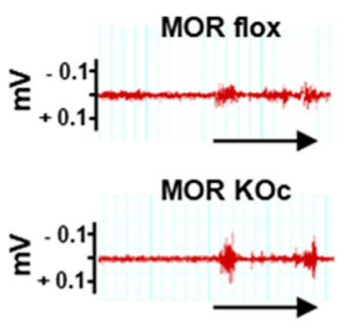

\section{C $\square$ MOR flox \\ MOR cKO}

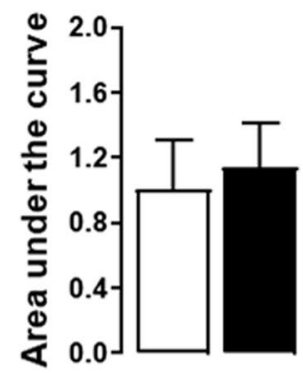

F

$\square$ DOR flox DOR cKO
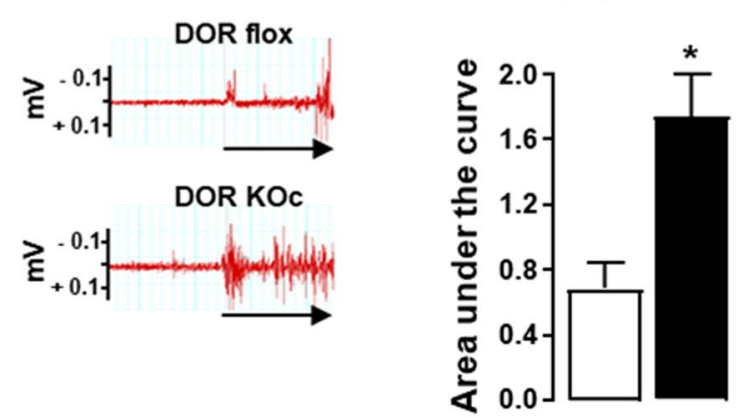

Fig. 5 DOR deletion in $\mathrm{Na}_{v}$ 1.8-expressing sensory neurons abolishes analgesia on day 12 of the DSS-induced colitis. Visceral sensitivity of conditional knockout mice (black symbols) for either MOR (MOR CKO) (A-C) or DOR (DOR cKO) (D-F) was compared to their corresponding littermate wild-type floxed mice (MOR flox and DOR flox) (white symbols) on day 12 of DSS treatment ( $n=5-7$ mice/group). A and $\mathbf{D}$ Visceromotor response (VMR) to colorectal distension. B and $\mathbf{E}$ Representative traces of abdominal muscle contractions in response to $60 \mathrm{~mm} \mathrm{Hg} \mathrm{colorectal}$ distension in littermate floxed mice and conditional $\mathrm{KO}$ mice. The arrows represent the $10 \mathrm{~s}$ of distension. $\mathbf{C}$ and $\mathbf{F}$ Area under the curve (AUC) calculated by plotting individual VMR. Data are expressed as mean \pm SEM. Statistical analysis was performed using repeated-measures two-way ANOVA and subsequent Sidak's multiple comparison tests (A and $\mathbf{D})$ or Mann-Whitney $U$ tests $(\mathbf{C}$ and $\mathbf{F}) .{ }^{*} p<0.05,{ }^{* *} p<0.01,{ }^{* * *} p<0.001$

small-diameter neurons that have properties of nociceptors while $23 \%$ co-express MOR and DOR [18]. We identified DOR expressed in DRG neurons as the main targets in the endogenous regulation of colitis-induced visceral pain by using DOR conditional knockout mice that display a selective inactivation of Oprd1 gene in small and medium size dorsal root ganglia (DRG) neurons while remaining active in the spinal cord and brain of the animals [16]. Our results obtained with a genetic approach are in line with a recent study showing that supernatants from colonic biopsies recovered from mice chronically treated with DSS inhibit nociceptor excitability via DOR but not MOR in vitro [22]. In these experiments, the inhibitory activity of colonic supernatants on the excitability of DRG neurons was prevented by antagonist for DOR but not MOR [22]. Of note, exogenous MOR agonist ligands were able to decrease neuronal excitability, showing that MOR is functional [22].

Both MOR and DOR are known to be locally upregulated in intestine under inflammatory conditions, a situation that could be responsible for the enhanced efficacy of agonist opioid ligands during colitis [31-33]. So, it has been shown that the MOR agonist DAMGO reduced excitability of nociceptive DRG neurons as assessed by patch clamp recording [44]. An observation recently substantiated, in the preclinical DSS-induced colitis model, by the peripheral analgesic effect of a novel MOR agonist preferentially active in the acidified environment of 
A

OMOR flox $\quad$ MOR cKO

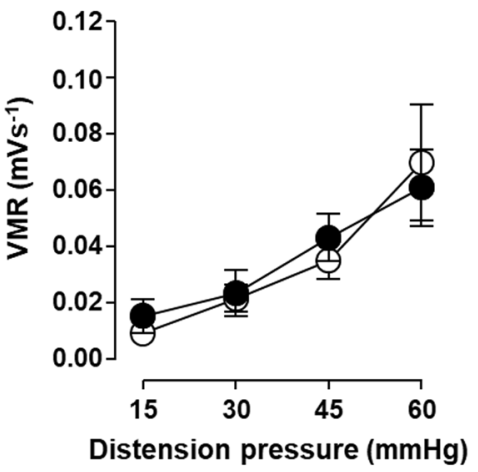

D

ODOR flox ODOR cKO

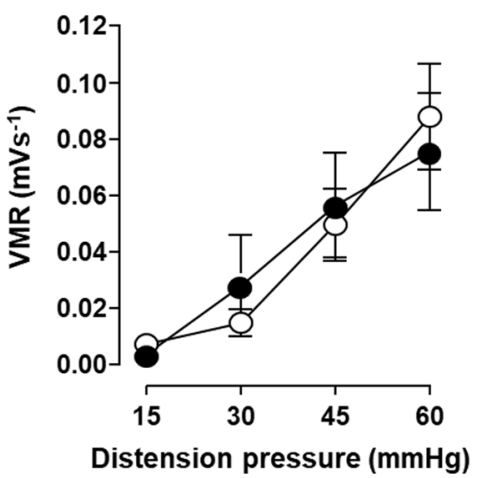

B

$\square$ MOR flox $\square$ MOR cKO

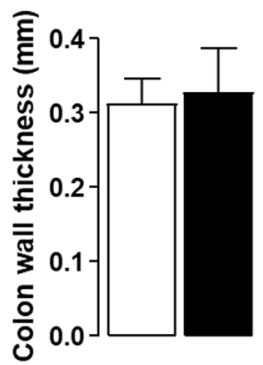

E

$\square$ DOR flox $\quad$ DOR cKO

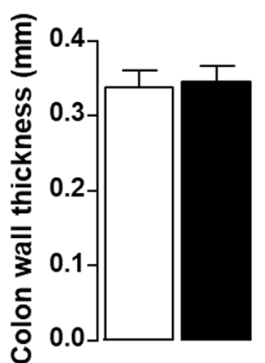

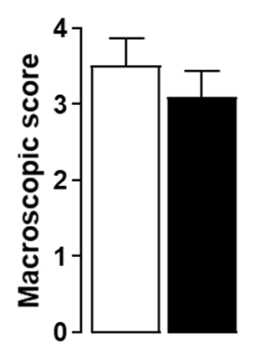

DDOR CKO

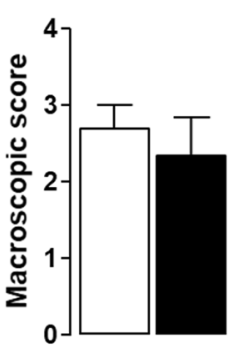

C

OMOR flox

- MOR cKO

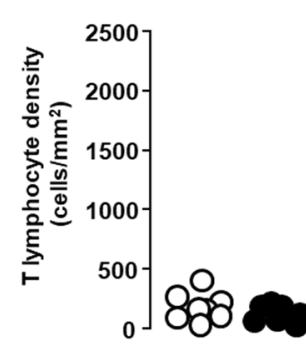

$\mathbf{F}$

ODOR flox

- DOR cKO

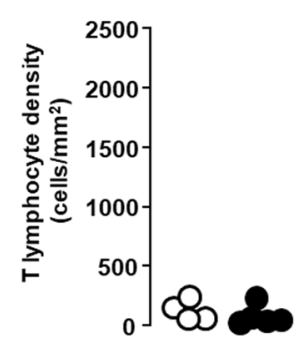

Fig. 6 Deletion of MOR or DOR in $\mathrm{Na}_{\mathrm{v}}$ 1.8-expressing sensory neurons does not impact inflammatory visceral pain in mice treated with $3 \%$ DSS for 5 days. Conditional knockout mice (black symbols) for either MOR (MOR CKO) (A-C) or DOR (DOR CKO) (D-F) were compared to their corresponding littermate wild-type floxed mice (MOR flox and DOR flox) (white symbols) ( $n=7-10$ mice/group) on day 5 of the 3\% DSS treatment for visceral sensitivity (A and $\mathbf{D})$, colitis severity assessed by colon wall thickness and macroscopic tissue damage $(\mathbf{B}$ and $\mathbf{E})$ and mucosal CD3 ${ }^{+} \mathrm{T}$ lymphocyte density (each point, corresponding to one animal, represents the mean of four different histological examinations per slice) $(\mathbf{C}$ and $\mathbf{F})(n=4-8$ mice/group). Data are expressed as mean \pm SEM. Statistical analysis was performed using repeated-measures two-way ANOVA (A and D) or MannWhitney $U$ test (B, C, E and $\mathbf{F})$

the inflamed colon [23]. Activation of DOR expressed on peripheral colonic nociceptors also induces potent analgesia in DSS-induced colitis model, endosomal signaling at DOR causing long-lasting inhibition of pain [22]. The beneficial effects of opioid agonists would also apply in the partial colon obstruction model as both analgesia and decrease in colon-specific DRG neuron excitability induced by Lactobacillus reuteri was reversed by the peripheral opioid receptor antagonist naloxone-methiodide [19].

Our observations indirectly corroborate the findings that mouse $\mathrm{T}$ lymphocytes mainly produce enkephalins, the most selective endogenous DOR ligands $[3,7,11]$ and that colitis is made painful when the enkephalin-encoding Penk gene is deleted in T lymphocytes [5, 9].
Moreover, as previously shown for somatic inflammatory pain [12], our study indicates that even if both MOR and DOR are expressed and functional in sensory neurons innervating the colon [16, 18, 21-23, 31, 48], only DOR is relevant for the endogenous $\mathrm{T}$ cell-mediated analgesia in mice as a $\mathrm{Na}_{\mathrm{v}} 1.8^{+}$neuron-expressed opioid receptor.

The ongoing opioid crisis leads to propose innovations in the management of visceral pain. Thus, to avoid centrally mediated side effects and a better benefit/risk ratio, a number of strategies strictly targeting opioid receptors in periphery are under investigation [23, 37, 41]. MOR that is the main target of pharmacological drugs commonly used in intestinal inflammatory disorders is also the source of a number of serious side effects such as 
bowel dysfunction. Because DOR activation produces similar but milder side effects, the pharmacological targeting of DOR appears potentially more attractive [17]. In this context, the key role of DOR on primary afferents in the endogenous control of intestinal inflammatory pain opens new therapeutic opportunities for peripherally restricted DOR analgesics including, as already developed for MOR agonists, molecules acting at low $\mathrm{pH}[15,41]$. Of note, the local production of endogenous opioids by immune cells within the inflamed colon might also reduce tolerance to chronic treatment by preserving DOR signaling in peripheral sensory neurons [50].

\section{Conclusions}

Taken together, our results suggest that the development of DOR agonists specifically active in periphery may improve effectiveness of opioid therapy in the treatment of chronic inflammatory visceral pain and dramatically reduce risk of developing addictive behavior.

\section{Abbreviations}

CKO: Conditional knockout; DRG: Dorsal root ganglia; MOR: $\mu$-Opioid receptor; DOR: $\delta$-Opioid receptor; DSS: Dextran sulfate sodium IBD: inflammatory bowel diseases.

\section{Acknowledgements}

The authors wish to thank ANEXPLO platforms (UMS 006) animal care facility (X. Collet and R. Balouzat), the platform Aninfimip, an EquipEx ('Equipement d'Excellence') supported by the French government through the Investments for the Future program (ANR-11-EQPX-0003), the histopathology core facility (F. Capilla) and the INFINITy cellular imaging core facility (S. Allart and S. Lachambre).

\section{Authors' contributions}

XM-O, LB, CB and NC performed the experiments. XM-O, LB, CGR, NC and GD designed and analyzed the data. GD supervised the study and wrote the manuscript. XM-O, LB, CGR, NC revised and approved the manuscript. All authors read and approved the final manuscript.

\section{Funding}

This work was supported by the Institut National de la Santé et de la Recherche Médicale (INSERM), the Université Paul Sabatier, Toulouse III; Université de Strasbourg and the French Agence Nationale de la Recherche grant LYMPHOPIOID.

\section{Availability of data and materials}

All data generated or analyzed during this study are included in this published article.

\section{Declarations}

\section{Ethics approval and consent to participate}

All procedures were performed in accordance with the Guide for the Care and Use of Laboratory Animals of the European Council and were approved by the Animal Care and Ethics Committee of US006/CREFE (CEEA-122; application number APAFIS \#16385-2018080222083660 v3).

\section{Consent for publication}

Not applicable.

\section{Competing interests}

The authors declare that they have no competing financial interests.

\section{Author details}

${ }^{1}$ Digestive Health Research Institute (IRSD), Université de Toulouse, INSERM, INRA, ENVT, UPS, CHU Purpan BP 3028, 31024 Toulouse Cedex 3, France. ${ }^{2}$ INFINITy, Université de Toulouse, INSERM, CNRS, UPS, Toulouse, France. ${ }^{3}$ IGBMC, Université de Strasbourg, INSERM, CNRS, IIIkirch, France.

Received: 26 April 2021 Accepted: 13 December 2021

Published online: 06 January 2022

\section{References}

1. Auge C, Basso L, Blanpied C, Vergnolle N, Game X, Chabot S, Lluel P, Dietrich G. Pain management in a model of Interstitial Cystitis/Bladder Pain Syndrome by a vaccinal strategy. Front Pain Res. 2021. https://doi. org/10.3389/fpain.2021.642706.

2. Baamonde A, Menendez L, Gonzalez-Rodriguez S, Lastra A, Seitz V, Stein C, Machelska H. A low pKa ligand inhibits cancer-associated pain in mice by activating peripheral mu-opioid receptors. Sci Rep. 2020;10(1):18599.

3. Baddack-Werncke U, Busch-Dienstfertig M, Gonzalez-Rodriguez S, Maddila SC, Grobe J, Lipp M, Stein C, Muller G. Cytotoxic T cells modulate inflammation and endogenous opioid analgesia in chronic arthritis. J Neuroinflammation. 2017;14(1):30.

4. Basso L, Benamar M, Mas-Orea X, Deraison C, Blanpied C, Cenac N, Saoudi A, Dietrich $G$. Endogenous control of inflammatory visceral pain by $T$ cell-derived opioids in IL-10-deficient mice. Neurogastroenterol Motil. 2020;32(2):e13743.

5. Basso L, Boue J, Auge C, Deraison C, Blanpied C, Cenac N, Lluel P, Vergnolle N, Dietrich G. Mobilization of CD4+T lymphocytes in inflamed mucosa reduces pain in colitis mice: toward a vaccinal strategy to alleviate inflammatory visceral pain. Pain. 2018;159(2):331-41.

6. Basso L, Boue J, Bourreille A, Dietrich G. Endogenous regulation of inflammatory pain by T-cell-derived opioids: when friend turns to foe. Inflamm Bowel Dis. 2014;20(10):1870-7.

7. Basso L, Boue J, Mahiddine K, Blanpied C, Robiou-du-Pont S, Vergnolle N, Deraison C, Dietrich G. Endogenous analgesia mediated by CD4(+)T lymphocytes is dependent on enkephalins in mice. J Neuroinflammation. 2016;13(1):132.

8. Basso L, Bourreille A, Dietrich G. Intestinal inflammation and pain management. Curr Opin Pharmacol. 2015;25:50-5.

9. Basso L, Garnier L, Bessac A, Boue J, Blanpied C, Cenac N, Laffont S, Dietrich G. T-lymphocyte-derived enkephalins reduce Th1/Th17 colitis and associated pain in mice. J Gastroenterol. 2018;53(2):215-26.

10. Boue J, Basso L, Cenac N, Blanpied C, Rolli-Derkinderen M, Neunlist $M$, Vergnolle N, Dietrich $G$. Endogenous regulation of visceral pain via production of opioids by colitogenic CD4 $(+)$ T cells in mice. Gastroenterology. 2014;146(1):166-75.

11. Boue J, Blanpied C, Brousset P, Vergnolle N, Dietrich G. Endogenous opioid-mediated analgesia is dependent on adaptive $T$ cell response in mice. J Immunol. 2011;186(9):5078-84.

12. Boue J, Blanpied C, Djata-Cabral M, Pelletier L, Vergnolle N, Dietrich G. Immune conditions associated with CD4+ T effector-induced opioid release and analgesia. Pain. 2012;153(2):485-93.

13. Burford NT, Traynor JR, Alt A. Positive allosteric modulators of the mu-opioid receptor: a novel approach for future pain medications. $\mathrm{Br} J$ Pharmacol. 2015;172(2):277-86.

14. Carbone SE, Poole DP. Inflammation without pain: Immune-derived opioids hold the key. Neurogastroenterol Motil. 2020;32(2):e13787.

15. Feng J, Lepetre-Mouelhi S, Gautier A, Mura S, Cailleau C, Coudore F, Hamon M, Couvreur P. A new painkiller nanomedicine to bypass the blood-brain barrier and the use of morphine. Sci Adv. 2019. https://doi. org/10.1126/sciadv.aau5148.

16. Gaveriaux-Ruff C, Nozaki C, Nadal X, Hever XC, Weibel R, Matifas A, Reiss D, Filliol D, Nassar MA, Wood JN, Maldonado R, Kieffer BL. Genetic ablation of delta opioid receptors in nociceptive sensory neurons increases chronic pain and abolishes opioid analgesia. Pain. 2011;152(6):1238-48.

17. Gendron L, Cahill CM, von Zastrow M, Schiller PW, Pineyro G. Molecular pharmacology of delta-opioid receptors. Pharmacol Rev. 2016;68(3):631-700

18. Guerrero-Alba R, Valdez-Morales EE, Jimenez-Vargas NN, Bron R, Poole D, Reed D, Castro J, Campaniello M, Hughes PA, Brierley SM, Bunnett N, 
Lomax AE, Vanner S. Co-expression of mu and delta opioid receptors by mouse colonic nociceptors. Br J Pharmacol. 2018;175(13):2622-34.

19. Hegde S, Lin YM, Fu Y, Savidge T, Shi XZ. Precision Lactobacillus reuteri therapy attenuates luminal distension-associated visceral hypersensitivity by inducing peripheral opioid receptors in the colon. Pain. 2020;161(12):2737-49.

20. Hua S, Cabot PJ. Targeted nanoparticles that mimic immune cells in pain control inducing analgesic and anti-inflammatory actions: a potential novel treatment of acute and chronic pain condition. Pain Physician. 2013;16(3):E199-216.

21. Hughes PA, Costello SP, Bryant RV, Andrews JM. Opioidergic effects on enteric and sensory nerves in the lower Gl tract: basic mechanisms and clinical implications. Am J Physiol Gastrointest Liver Physiol. 2016;311(3):G501-513

22. Jimenez-Vargas NN, Gong J, Wisdom MJ, Jensen DD, Latorre R, Hegron A, Teng S, DiCello JJ, Rajasekhar P, Veldhuis NA, Carbone SE, Yu Y, LopezLopez C, Jaramillo-Polanco J, Canals M, Reed DE, Lomax AE, Schmidt BL, Leong KW, Vanner SJ, Halls ML, Bunnett NW, Poole DP. Endosomal signaling of delta opioid receptors is an endogenous mechanism and therapeutic target for relief from inflammatory pain. Proc Natl Acad Sci U S A. 2020;117(26):15281-92.

23. Jimenez-Vargas NN, Yu Y, Jensen DD, Bok DD, Wisdom M, Latorre R, Lopez C, Jaramillo-Polanco JO, Degro C, Guzman-Rodriguez M, Tsang Q, Snow Z, Schmidt BL, Reed DE, Lomax AE, Margolis KG, Stein C, Bunnett NW, Vanner SJ. Agonist that activates the micro-opioid receptor in acidified microenvironments inhibits colitis pain without side effects. Gut. 2021. https://doi.org/10.1136/gutjnl-2021-324070.

24. Kavelaars A, Heijnen CJ. T cells as guardians of pain resolution. Trends Mol Med. 2021;27(4):302-13.

25. Mace G, Blanpied C, Emorine L, Druet P, Dietrich G. Morphine-like activity of natural human lgG autoantibodies is because of binding to the first and third extracellular loops of the mu-opioid receptor. J Biol Chem. 1999;274(29):20079-82.

26. Machelska H, Celik MO. Advances in achieving opioid analgesia without side effects. Front Pharmacol. 2018;9:1388.

27. Majumdar S, Grinnell S, Le Rouzic V, Burgman M, Polikar L, Ansonoff M, Pintar J, Pan YX, Pasternak GW. Truncated G protein-coupled mu opioid receptor MOR-1 splice variants are targets for highly potent opioid analgesics lacking side effects. Proc Natl Acad Sci U S A. 2011;108(49):19778-83.

28. Manglik A, Lin H, Aryal DK, McCorvy JD, Dengler D, Corder G, Levit A, Kling RC, Bernat V, Hubner H, Huang XP, Sassano MF, Giquere PM, Lober S, Da D, Scherrer G, Kobilka BK, Gmeiner P, Roth BL, Shoichet BK. Structurebased discovery of opioid analgesics with reduced side effects. Nature. 2016;537(7619):185-90.

29. Nassar MA, Stirling LC, Forlani G, Baker MD, Matthews EA, Dickenson AH, Wood JN. Nociceptor-specific gene deletion reveals a major role for Nav1.7 (PN1) in acute and inflammatory pain. Proc Natl Acad Sci U S A. 2004;101(34):12706-11.

30. Niccum B, Moninuola O, Miller K, Khalili H. Opioid use among patients with inflammatory bowel disease: a systematic review and meta-analysis. Clin Gastroenterol Hepatol. 2021;19(5):895-907.e894.

31. Philippe D, Dubuquoy L, Groux H, Brun V, Chuoi-Mariot MT, GaveriauxRuff C, Colombel JF, Kieffer BL, Desreumaux P. Anti-inflammatory properties of the mu opioid receptor support its use in the treatment of colon inflammation. J Clin Invest. 2003;111(9):1329-38.

32. Pol O, Alameda F, Puig MM. Inflammation enhances mu-opioid receptor transcription and expression in mice intestine. Mol Pharmacol. 2001;60(5):894-9.

33. Pol O, Palacio JR, Puig MM. The expression of delta- and kappa-opioid receptor is enhanced during intestinal inflammation in mice. J Pharmacol Exp Ther. 2003;306(2):455-62.

34. Pujo J, Petitfils C, Le Faouder P, Eeckhaut V, Payros G, Maurel S, PerezBerezo T, Van Hul M, Barreau F, Blanpied C, Chavanas S, Van Immerseel F, Bertrand-Michel J, Oswald E, Knauf C, Dietrich G, Cani PD, Cenac N. Bacteria-derived long chain fatty acid exhibits anti-inflammatory properties in colitis. Gut. 2021;70(6):1088-97.

35. Reaux-Le Goazigo A, Poras H, Ben-Dhaou C, Ouimet T, Baudouin C, Wurm M, Melik PS. Dual enkephalinase inhibitor PL265: a novel topical treatment to alleviate corneal pain and inflammation. Pain. 2019;160(2):307-21
36. Reiss D, Ceredig RA, Secher T, Boue J, Barreau F, Dietrich G, Gaveriaux-Ruff C. Mu and delta opioid receptor knockout mice show increased colonic sensitivity. Eur J Pain. 2017;21(4):623-34.

37. Rodriguez-Gaztelumendi A, Spahn V, Labuz D, Machelska H, Stein C. Analgesic effects of a novel $\mathrm{pH}$-dependent mu-opioid receptor agonist in models of neuropathic and abdominal pain. Pain. 2018;159(11):2277-84.

38. Schmid CL, Kennedy NM, Ross NC, Lovell KM, Yue Z, Morgenweck J, Cameron MD, Bannister TD, Bohn LM. Bias factor and therapeutic window correlate to predict safer opioid analgesics. Cell. 2017;171(5):1165-1175. e1113.

39. Soelberg CD, Brown RE Jr, Du Vivier D, Meyer JE, Ramachandran BK. The US opioid crisis: current federal and state legal issues. Anesth Analg. 2017:125(5):1675-81.

40. Spahn V, Del Vecchio G, Labuz D, Rodriguez-Gaztelumendi A, Massaly N, Temp J, Durmaz V, Sabri P, Reidelbach M, Machelska H, Weber M, Stein C. A nontoxic pain killer designed by modeling of pathological receptor conformations. Science. 2017;355(6328):966-9.

41. Stein C. New concepts in opioid analgesia. Expert Opin Investig Drugs. 2018;27(10):765-75

42. Stirling LC, Forlani G, Baker MD, Wood JN, Matthews EA, Dickenson AH, Nassar MA. Nociceptor-specific gene deletion using heterozygous NaV1.8-Cre recombinase mice. Pain. 2005;113(1-2):27-36.

43. Targownik LE, Nugent Z, Singh H, Bugden S, Bernstein CN. The prevalence and predictors of opioid use in inflammatory bowel disease: a population-based analysis. Am J Gastroenterol. 2014;109(10):1613-20

44. Valdez-Morales E, Guerrero-Alba R, Ochoa-Cortes F, Benson J, Spreadbury I, Hurlbut D, Miranda-Morales M, Lomax AE, Vanner S. Release of endogenous opioids during a chronic IBD model suppresses the excitability of colonic DRG neurons. Neurogastroenterol Motil. 2013;25(1):39-46.e34.

45. Verma-Gandhu M, Bercik P, Motomura Y, Verdu EF, Khan WI, Blennerhassett PA, Wang L, El-Sharkawy RT, Collins SM. CD4+ T-cell modulation of visceral nociception in mice. Gastroenterology. 2006;130(6):1721-8.

46. Verma-Gandhu M, Verdu EF, Bercik P, Blennerhassett PA, Al-Mutawaly N, Ghia JE, Collins SM. Visceral pain perception is determined by the duration of colitis and associated neuropeptide expression in the mouse. Gut. 2007;56(3):358-64.

47. Vowles KE, McEntee ML, Julnes PS, Frohe T, Ney JP, van der Goes DN. Rates of opioid misuse, abuse, and addiction in chronic pain: a systematic review and data synthesis. Pain. 2015;156(4):569-76.

48. Weibel R, Reiss D, Karchewski L, Gardon O, Matifas A, Filliol D, Becker JA, Wood JN, Kieffer BL, Gaveriaux-Ruff C. Mu opioid receptors on primary afferent nav 1.8 neurons contribute to opiate-induced analgesia: insight from conditional knockout mice. PLoS ONE. 2013;8(9):e74706.

49. Zhou X, Qiao G, Ren J, Wang X, Wang S, Zhu S, Yuan Y, Morse MA, Hobeika A, Lyerly HK. Adoptive immunotherapy with autologous T-cell infusions reduces opioid requirements in advanced cancer patients. Pain. 2020;161(1):127-34.

50. Zollner C, Mousa SA, Fischer O, Rittner HL, Shaqura M, Brack A, Shakibaei M, Binder W, Urban F, Stein C, Schafer M. Chronic morphine use does not induce peripheral tolerance in a rat model of inflammatory pain. J Clin Invest. 2008;118(3):1065-73.

\section{Publisher's Note}

Springer Nature remains neutral with regard to jurisdictional claims in published maps and institutional affiliations. 